\title{
Disappearing breast cysts
}

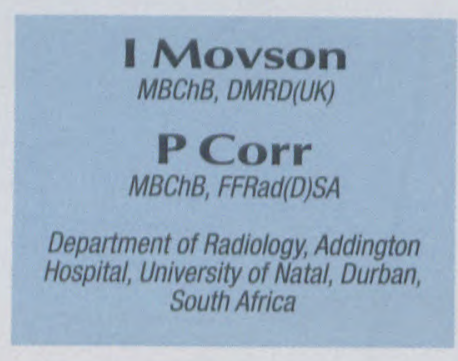

\section{Abstract}

The natural history of simple breast cysts is illustrated by our patient with multiple palpable cysts which disappeared completely over a six month period.

\section{Interval}

mammography is all that is required in these patients.

\section{Case report}

A 52 year-old woman was referred for a mammogram because of a palpable mass in the outer quadrant of the right breast. Mammography (Figure 1) demonstrated multiple, well defined masses bilaterally. There was no microcalcification or spicultation. Ultrasound confirmed that these were simple cysts. Cyst puncture was not

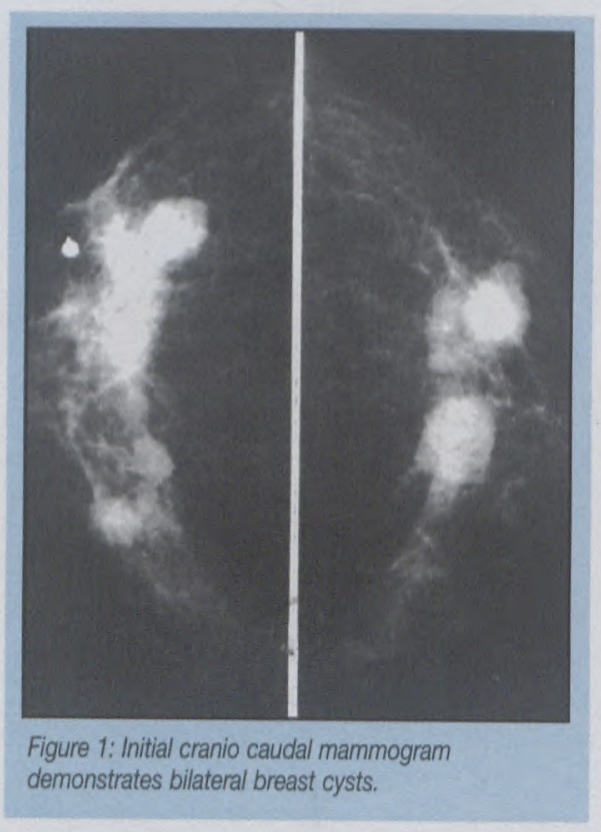

performed. A repeat mammogram was advised after six months. This demonstrated that the previous cysts had resolved completely (Figure 2).

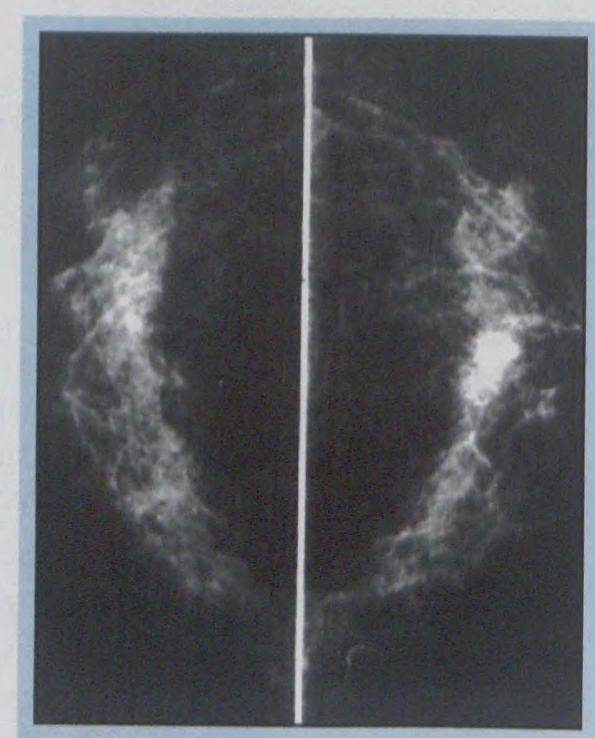

Figure 2: Follow-up mammogram after a six month interval confirms disappearance of the cysts.

\section{Discussion}

Breast cysts are common, occurring in $30-50 \%$ of women at autopsy. ${ }^{1}$ The peak incidence is between $40-49$ years of age. The reason why cysts disappear is unknown but is probably due to changing oestrogen levels as they frequently regress after menopause. Our patient is unusual as the cysts disappeared so rapidly.

Screening programmes have demonstrated that more than $50 \%$ of interval cysts resolve within one year and by two years, two-thirds had disappeared. At five years less than $12 \%$ of cysts were unchanged. ${ }^{2}$

The cause of simple breast cysts is unclear. They originate from the terminal ductule lobular unit. They are thought to result from coalescence of lobular acini during ductule involution or from ductule obstruction. The cyst wall is composed of cuboidal to columnar epithelium which is often multilayered but can be atrophic. ${ }^{3}$ Occasionally cysts develop following duct ectasia, fat necrosis, or oestrogen administration. ${ }^{4}$

Patients are often asymptomatic although pain and tenderness may occur from fluid tension in the cysts. The cysts vary in size with the menstrual cycle. ${ }^{4}$

On mammography, breast cysts are indistinguishable from other benign masses especially fibroadenomas - however ultrasound confirmation of a simple cyst establishes the diagnosis.

Interval annual mammography is all that is required in these patients to confirm cyst regression.

\section{References}

1. Kopans D. Breast Imaging. Philadelphia: Lipincott. 1989; 266-267.

2. Brenner RJ, Bein ME, Sarti DA, Vinstein AL. Spontaneous regression of interval benign cysts of the breast. Radiology, 1994; 193:365-368.

3. Haagensen CD. Disease of the breast. WB Saunders, Philadelphia 1986

4. Tucker AK. Textbook of mammography. Churchill Livingstone, Edinburgh, 1993; 120-167. 